\title{
Intermolecular Interactions in Organic Dye Monolayers Formed by Langmuir-Blodgett Technique
}

\begin{abstract}
D. BAUMAN*
Faculty of Technical Physics, Poznań University of Technology, Nieszawska 13A, 60-965 Poznań, Poland

Langmuir and Langmuir-Blodgett films of some organic dyes (perylene-like dyes, derivatives of 4-aminonaphthalimide, derivatives of naphthoylenebenzimidazole, azo dyes) and their mixtures with thermotropic liquid crystal or arachidic acid have been investigated. Surface pressure as a function of the mean molecular area for the Langmuir films were measured and information about organization of molecules at the air-water interface has been obtained. Absorption spectra in situ for the Langmuir films and both absorption and fluorescence spectra for Langmuir-Blodgett films were recorded. The results obtained from spectroscopic studies have led to conclusions about formation of self-aggregates of dye molecules, both in ground and excited states, at the phases interfaces.
\end{abstract}

PACS numbers: 68.18.-g, 68.47.Pe, 78.40.Me, 78.55.Kz

\section{Introduction}

The progress in the miniaturization of electronic devices causes the necessity to use in them single molecules or small molecular aggregates. Various nanotechnologies can be here helpful - we search for quick, reliable and economically efficient methods which will produce systems on the nanoscale. The Langmuir-Blodgett (LB) technique [1] is one of such methods. It is a unique method which allows us to fabricate two-dimensional (2D) ordered layers from some molecules and colloid particles, the architecture of which can be manipulated easily. This gives possibility to optimize the physical (especially electro-optical) parameters of a material used and therefore the LB films are today the integral part of the knowledge area connected with the molecular electronics. The LB technique was also proposed to obtain the ordered layer for organic light emitting diodes (OLEDs) [2], which constitute today the largest competition with respect to liquid crystal displays (LCDs).

On the other hand, the fabrication of ultrathin layers by LB technique is very useful to study molecular aggregates, especially of organic compound molecules. These layers give the molecules the opportunity to be in highly oriented environments, similarly as it takes place in solid matrices, where the phenomenon of the molecular aggregation frequently occurs [3]. In the recent years, molecular aggregates have attracted a considerable attention because of very interesting optical properties of these systems and their possible application as novel functionalized materials in molecular electronics and photonics [4].
In order to fabricate LB films it is necessary to create first the compressible and stable monolayer at the airwater interface (the Langmuir film) [1]. The Langmuir films are usually formed by amphiphilic molecules, i.e. molecules possessing distinct hydrophilic and hydrophobic parts, and the most well known compounds are here fatty acids. Thermotropic liquid crystal molecules cannot be directly treated as amphiphilic, nevertheless many of them are able to create stable compressible monolayers at the liquid surface, which can be next transferred at the solid substrates. Already several years ago it was found that some of thermotropic liquid crystals with rod-like shape, whose molecules possess strongly polar hydrophilic head group and hydrophobic alkyl chain, can form stable two-dimensional monolayers at the air- water interface, which can be transferred onto the solid substrates making LB films [5]. Moreover, there are other molecules which, despite being non-amphiphilic, can form stable Langmuir films [1, 6].

This review article presents the results of the study of the LB films formed of some organic dyes (perylene-like dyes, derivatives of 4-aminonaphthalimide, derivatives of naphthoylenebenzimidazole, azo dyes) and of their mixtures with thermotropic liquid crystal or arachidic acid. The aim of the study was to determine the intermolecular interactions leading to formation of various kinds of aggregates, both in ground and excited electronic states, in ultrathin ordered films.

* e-mail: danuta.bauman@put.poznan.pl 


\section{Materials and methods}

\subsection{Materials}

The following dyes were investigated:

1. 3,4,9,10-tetra-(n-alkoxy-carbonyl)-perylenes

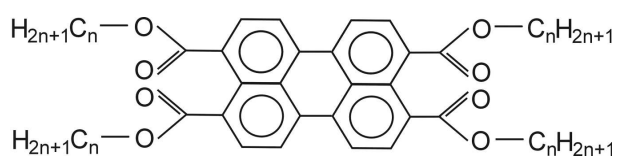

\begin{tabular}{c|c}
\hline \hline Dye code & $n$ \\
\hline P1 & 3 \\
P2 & 4 \\
P3 & 5 \\
P4 & 6 \\
P5 & 7 \\
P6 & 8
\end{tabular}

2. derivatives of 4-aminonaphthalimide

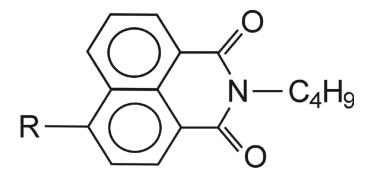

\begin{tabular}{c|c}
\hline \hline Dye code & $R$ \\
\hline AN1 & \\
AN2 & \\
AN3 & \\
AN4 &
\end{tabular}

3. derivatives of naphthoylenebenzimidazole

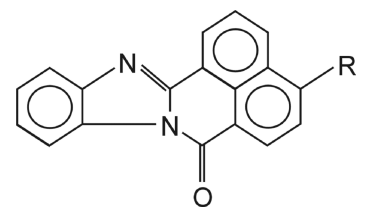

\begin{tabular}{c|c}
\hline \hline Dye code & $\mathrm{R}$ \\
\hline $\mathrm{NB} 1$ & $-\mathrm{NH}\left(\mathrm{CH}_{2}\right)_{2} \mathrm{CH}_{3}$ \\
$\mathrm{NB} 2$ & $-\mathrm{N}\left(\mathrm{CH}_{3}\right)_{2}$ \\
$\mathrm{NB} 3$ &
\end{tabular}

4. azo dyes

\begin{tabular}{|c|c|}
\hline Dye code & Molecular structure \\
\hline AZO1 & \\
\hline $\mathrm{AZO} 2$ & \\
\hline AZO3 & \\
\hline $\mathrm{AZO} 4$ & \\
\hline AZO5 & \\
\hline AZO6 & \\
\hline $\mathrm{AZO} 7$ & \\
\hline
\end{tabular}

All the dyes were synthesized and chromatographically purified at the Institute of Polymer Technology and Dyes, Łódź University of Technology, Poland.

Two thermotropic liquid crystals: 4-heptyl-4'-cyanobiphenyl (7CB) and 4-octyl-4'-cyanobiphenyl (8CB) as well as the fatty acid $\mathrm{C}_{20} \mathrm{H}_{40} \mathrm{O}_{2}$ (arachidic acid - AA) were used as supporting matrices. The liquid crystals chosen are known as compounds that form stable Langmuir and LB films [5]. They were purchased from Prof. R. Dąbrowski Laboratory at the Military University of Technology, Warsaw (Poland) and were used without further purification. Arachidic acid (AA) with a quoted purity of $>99 \%$ was obtained from Sigma-Aldrich.

\subsection{Methods}

Monolayers at the air-water (Langmuir films) and airsolid substrate (LB films) interfaces were prepared using Minitrough 2 (KSV Instruments Ltd., Finland) equipped with two barriers for monolayer compression and a Wilhelmy plate balance for surface pressure determination (Fig. 1). Milli-Q water was used as a subphase and its temperature was maintained by cooling circulator and kept constant at $(20.5 \pm 0.5)^{\circ} \mathrm{C}$. Polished quartz plates were used as solid substrates with a hydrophilic surface. The vertical dipping method (Fig. 2a) was used to obtain LB films. Successful deposition of pure dyes and dye/liquid crystal mixtures took place only by the first raising of the substrate. The deposition of the floating layer of dye/AA mixtures was possible at repeated dipping and multilayers of both $Y$ and $Z$ types (Fig. 2b) were fabricated. The transfer rate was $5 \mathrm{~mm} / \mathrm{min}$, and the transfer ratio [6] was between 1.00 and 1.20. Further experimental details are given in Refs. [7-11].

The absorption spectra of LB films were recorded in the UV-Vis region by means of a spectrophotometer CARY 400, while the fluorescence measurements were 


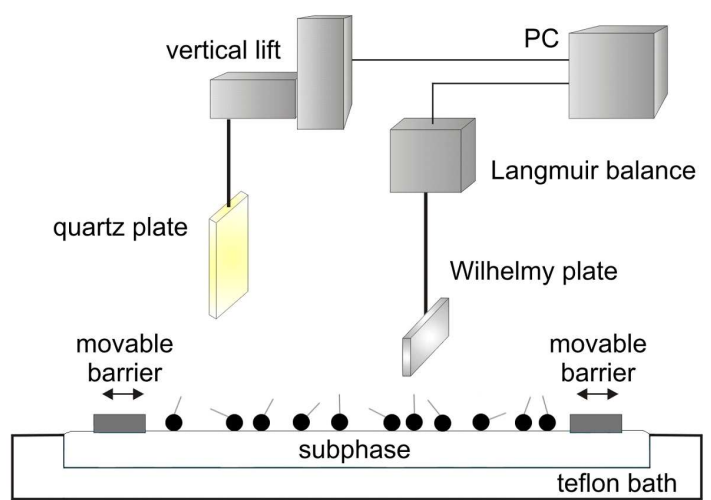

Fig. 1. Trough for Langmuir and Langmuir-Blodgett films preparation.

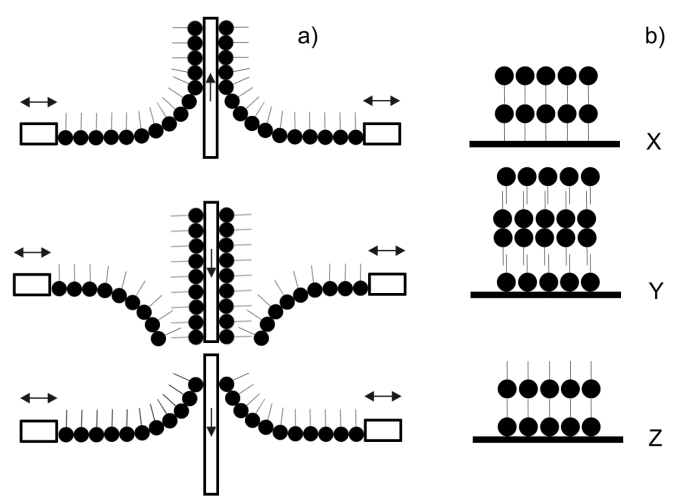

Fig. 2. (a) Deposition of the floating monolayer onto a solid substrate. (b) Different multilayer structures possible in LB films.

carried out by using a photon-counting spectrofluorimeter built in our laboratory and described in detail in [12]. The exciting light was $436 \mathrm{~nm}$ line from high-pressure mercury lamp. Additionally, absorption spectra of spread monolayers on the water surface were recorded in situ using fiber optic accessory supplied by Varian and connected with spectrophotometer. The details of the equipment are described in [13].

\section{Results and discussion}

\subsection{Molecular organization in Langmuir films}

All 3,4,9,10-tetra-( $n$-alkoxy-carbonyl)-perylenes form compressible and stable monolayers on the water surface. Among derivatives of 4-aminonaphthalimide and naphthoylenebenzimidazole, only dyes AN5 and NB3 which have the morpholine ring in the molecular structure, are not able to create Langmuir films. It was also not possible to fabricate Langmuir films from any of azo dyes, although the molecules of these dyes possess terminal polar $-\mathrm{NO}_{2}$ group. However, all the dyes under investigation can form compressible monolayers when they are mixed with one of cyanobiphenyls or with the arachidic acid.

A basic and widely used technique to characterize a Langmuir film is the measurement of the surface pressure as a function of the mean area available for one molecule $(\pi-A$ isotherm). Such an isotherm is rich in information on stability of the monolayer, the organization of molecules at the air-water interface, phase transitions, and conformational transformations. For compounds under investigation $\pi-A$ isotherm was recorded both at compression and expansion processes. The stable monolayers showed only small hysteresis.

Figures 3 and 4 present exemplary $\pi-A$ isotherms of the Langmuir films formed of, respectively, derivatives of 4-aminonaphthalimide and mixtures of dye NB2 with the arachidic acid at various dye molar fraction, $x_{\mathrm{M}}$. The shape of $\pi-A$ isotherms depends on the molecular structure of the dye, and, in the case of mixtures, on their composition. For all compressible monolayers the increase of $\pi$ begins at the area equal to $A_{0}$ and rises monotonically up to the collapse point, determined by the values $A_{\mathrm{C}}$ and $\pi_{\mathrm{C}}$ (see inset in Fig. 3). By further restriction of the available area, the less or more distinct plateau is observed. The $\pi_{\mathrm{C}}$ value gives information about the monolayer stability: the higher $\pi_{\mathrm{C}}$ means the better stability, while from the steepness of the $\pi-A$ isotherms the conclusions about the monolayer rigidity can be drawn. The value of $A_{\mathrm{C}}$ allows us to determine the packing of molecules in the Langmuir film as well as to calculate the tilt angle of the molecular long axis with respect to the normal to the water surface. The molecules of all the dyes create some angle with the normal, but it is significantly smaller than the tilt angle formed by the liquid crystal molecules being about $60^{\circ}$. Therefore, the dyes can "stiffen" the Langmuir films of cyanobiphenyls.

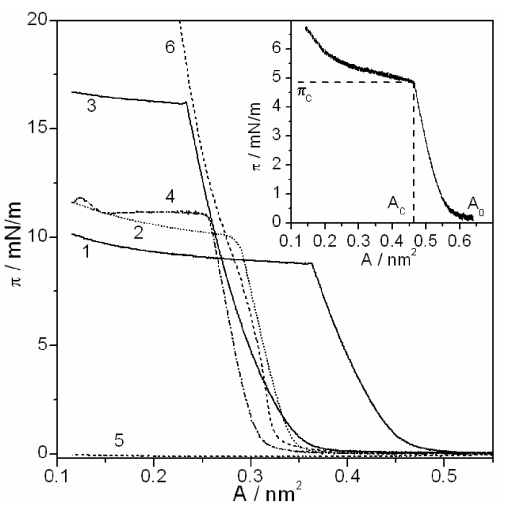

Fig. 3. Surface pressure-mean molecular area $(\pi-A)$ isotherms of the Langmuir films of derivatives of 4-aminonaphthalimide: AN1 (1), AN2 (2), AN3 (3), AN4 (4), AN5 (5) and AN6 (6) [9]. Inset description is given in the text.

The $\pi-A$ isotherms of monolayers formed by binary mixtures enable to determine the miscibility or phase separation of both components. In order to make this, the 


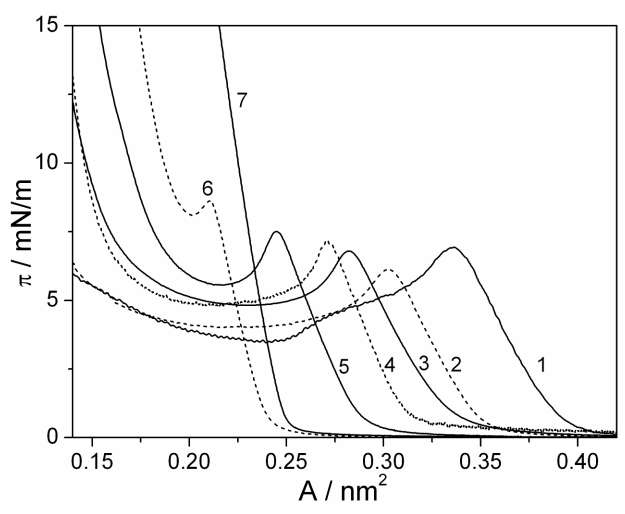

Fig. 4. Surface pressure-mean molecular area $(\pi-A)$ isotherms of Langmuir films of dye NB2 (1) and NB2/AA mixtures with $x_{\mathrm{M}}$ of 0.8 (2), 0.6 (3), 0.5 (4), $0.4(5), 0.2(6)$ and $0.0(7)$ [10].

deviation of the mean molecular area from the additivity rule, i.e. the excess area, as well as the dependence of $\pi_{\mathrm{C}}$ value on the dye molar fraction should be analysed [6]. The detailed results concerning the components miscibility and other parameters characterizing the Langmuir films of the dyes under investigation and of their mixtures with $7 \mathrm{CB}, 8 \mathrm{CB}$ or $\mathrm{AA}$ are presented in Refs. [7-11].

\subsection{Aggregation of dye molecules in ground electronic state}

The absorption spectra of the dyes and their mixtures with the liquid crystal or the arachidic acid were recorded both for the Langmuir and LB films. Figure 5 shows the absorption spectrum of P5/AA mixture at $x_{\mathrm{M}}=0.5$ in three-layer LB film and, for comparison, the spectrum of P5 dissolved in ethanol at $x_{\mathrm{M}}=1.5 \times 10^{-7}$. The large difference in the shape of both spectra is seen: the maxima are shifted, the spectrum of the LB film is significantly broadened. Similar results for other perylene-like dyes and for these dyes in the Langmuir films were obtained [7]. Although for other dyes under investigation no such large differences in the shape of the spectra recorded in diluted solutions (ethanol or chloroform) and in the Langmuir and LB films were found, in all the cases the changes of the maximum position and of the half-bandwidth were observed [8-11].

The absorption band of the dyes in the Langmuir and LB films was always strongly broadened with respect to the band recorded for the dyes in isotropic solvents and in the liquid crystals at small concentration (up to $x_{\mathrm{M}} \approx 10^{-3}$ ). This is illustrated for two azo dyes in Fig. 6 . When the dye content increased, the absorption band became broader and broader. The position of the absorption maxima of 3,4,9,10-tetra-( $n$-alkoxy-carbonyl)-perylenes as well as derivatives of 4-aminonaphthalimide and naphthoylenebenzimidazole in the Langmuir and LB films were shifted toward longer wavelengths with respect to the maximum position in the solution. In the case of azo dyes, the direction of the maximum shift was depen-

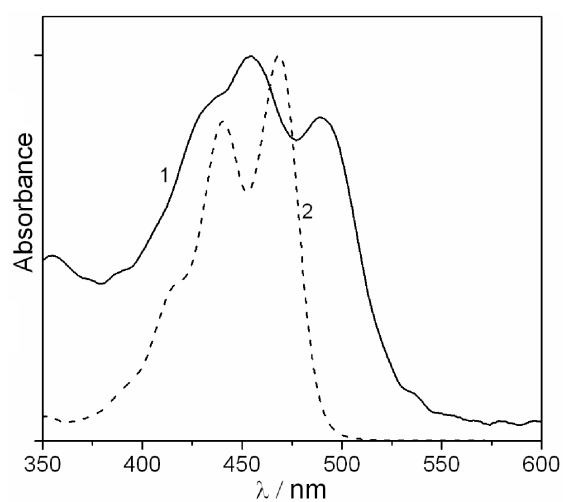

Fig. 5. Normalized long-wavelength absorption spectra (in the visible spectral region) of $\mathrm{P} 5 / \mathrm{AA}$ mixture with $x_{\mathrm{M}}=0.5$ in LB film of 3 layers deposited on quartz slide at $\pi=9 \mathrm{mN} / \mathrm{m}$ (1) and P5 in ethanol with $x_{\mathrm{M}}=1.5 \times 10^{-7}$ (2) [7].

dent on the content of the dye in the film: at small $x_{\mathrm{M}}$ the bathochromic shift was observed, and with increasing $x_{\mathrm{M}}$ the maximum was shifted back towards shorter wavelengths. The low concentration of the dyes in solutions ( $x_{\mathrm{M}}$ of order of $10^{-7}-10^{-3}$ ) suggests that we are dealing with the monomeric absorption. The shift and the broadening of the absorption bands in the Langmuir and LB films, where the dye concentration is significantly higher, indicate on the creation of the self-aggregates by the dye molecules in the ultrathin layers at the interfaces.

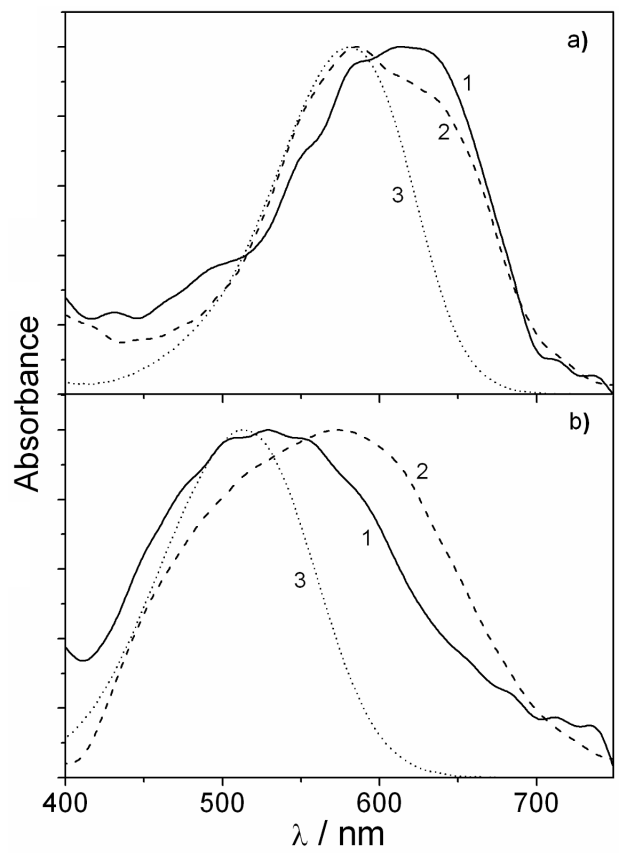

Fig. 6. Normalized long-wavelength absorption spectra of Langmuir (1) and LB (2) films of AZO1/8CB (a) and $\mathrm{AZO} 4 / 8 \mathrm{CB}(\mathrm{b})$ mixtures with $x_{\mathrm{M}}=0.5$. Curves 3 present absorption spectra of dyes dissolved in $8 \mathrm{CB}$ at $x_{\mathrm{M}}=10^{-3}[8]$. 
Analysis of the results obtained was made on the basis of the molecular exciton model [14]. According to this model, the interaction between transition dipoles of molecules in the aggregate results in the splitting of the energy level of the excited state into two levels with higher and lower energy relative to the undisturbed excited state (the Davydov splitting). Assuming the parallel configuration of dyes molecules in the simplest aggregate (dimer), the co-planar arrangement of the absorption transition moments can be considered. In this case the transition to one of the excited states, corresponding to an antiparallel alignment of dipole moments, is forbidden, while the energy difference between the excited monomer state and the exciton state depends on the angle $\theta$ between the dipole transition moment of the molecule and the vector joining the centers of two dipoles. When the alignment of the dipole moments in the aggregates is such that $0^{\circ}<\theta<54.7^{\circ}$, the exciton band is located energetically below the monomer band causing a bathochromic shift of the absorption spectrum, and created aggregates are called $J$-aggregates [15]. For $54.7^{\circ}<\theta<90^{\circ}$, the exciton band is located energetically above the monomer band causing a hypsochromic shift, and corresponding aggregates are referred to as $H$-aggregates [15]. When $\theta \approx 54.7^{\circ}$, no shift in the absorption spectrum is observed, and the aggregates are then called I-aggregates [16].

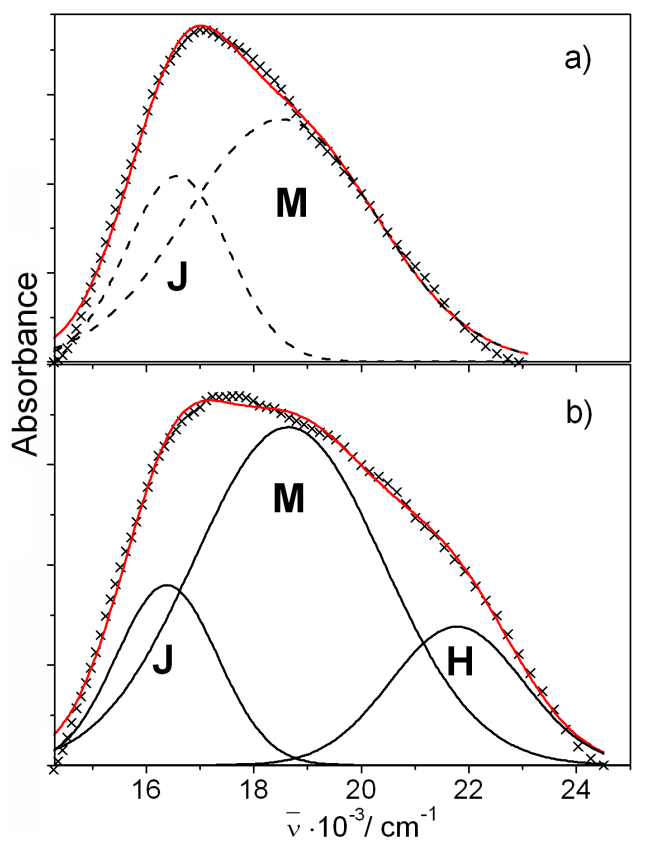

Fig. 7. Decomposition of the absorption spectrum of LB films of AZO4/8CB mixtures with $x_{\mathrm{M}}$ of 0.1 (a) and 0.5 (b) into Gaussian-type absorption bands [8].

The red shift of the absorption band observed for the most dyes investigated indicates the creation of some fraction of the $J$-type aggregates in the Langmuir and LB films. In the case of the derivatives of the naphthoylenebenzimidazole this shift is rather small, which suggests that they form also the $I$-type aggregates. However, the azo dyes, besides of $J$-type aggregates can create at higher concentrations also the aggregates of $H$-type. For the mixtures of the later dyes with $8 \mathrm{CB}$ in the LB films an attempt was made to separate the absorption spectra into two or three bands, corresponding to the absorption of monomers, $J$ - and/or $H$-aggregates. The values of $\lambda$ and $\delta$ of the absorption band of the dyes in $8 \mathrm{CB}$ at $x_{\mathrm{M}}=10^{-3}$ were taken as the fitting parameters for the monomer (M) absorption band. Next, one or two additional maxima were assumed, which should appear at the longer and/or shorter wavelengths with respect to the peak position of the monomer. A sum of normalized Gaussians was used as a model function for the experimentally obtained absorption band of azo dye/liquid crystal mixtures in the LB films. The exemplary results are shown in Fig. 7. It is seen that at low concentration, the absorption spectrum consists of the overlapped bands of monomers (M) and $J$-type aggregates (Fig. 7a). When the concentration rises, in the absorption spectrum the contribution of the $H$-type aggregates appears, too (Fig. 7b).

The results obtained from the absorption study indicate that all the dyes under investigation are able to creation of self-aggregates already in the ground electronic state. The detailed data and their analysis are presented in Refs. [7-11].

\subsection{Aggregation of dye molecules in excited state}

The fluorescence spectra were recorded for $3,4,9,10$ -tetra-( $n$-alkoxy-carbonyl)-perylenes, derivatives of 4-aminonaphthalimide, derivatives of naphthoylenebenzimidazole and their mixtures with the liquid crystal or the arachidic acid in the LB films. Figure 8 shows the fluorescence spectrum of P5/AA mixture at $x_{\mathrm{M}}=0.5$ in three-layer LB film and, for comparison, the spectrum of $\mathrm{P} 5$ dissolved in ethanol at $x_{\mathrm{M}}=1.5 \times 10^{-7}$. The spectrum of the LB film differs significantly from that in diluted solution: the structureless broad fluorescence band with significantly shifted $(\approx 100 \mathrm{~nm})$ maximum position is observed. Similar spectra were obtained for other perylene-like dyes, with the red shift of the maximum dependent on the alkyl chains length. As the so strongly shifted fluorescence band has no equivalence in the absorption spectrum, one can assume that we deal here with the aggregates occurring only in the excited state. It was supposed that the main emitters in the LB films are excimers, which originate from interaction of neighbouring molecules after one is excited [3]. The excimer formation is often observed in the solid phase of flat aromatic molecules and at their high concentrations in solutions $[3,17]$. In these cases, the exciton is delocalized over several molecules due to the large intermolecular interactions. Therefore, the excimer creation was observed also in the LB films, where the high dye concentration and molecular ordering favour the aggregation process $[12,18,19]$. 


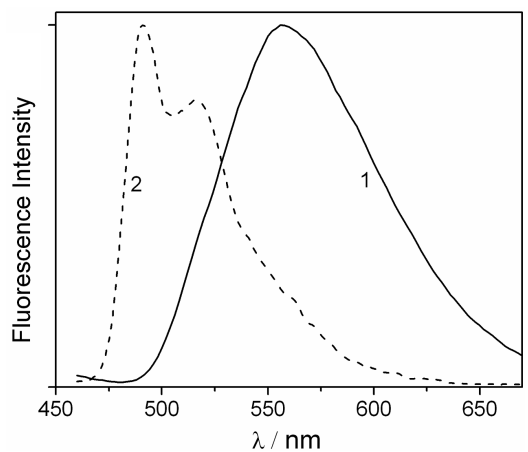

Fig. 8. Normalized fluorescence spectra of P5/AA mixture with $x_{\mathrm{M}}=0.5$ in LB film of 3 layers deposited on quartz slide at $\pi=9 \mathrm{mN} / \mathrm{m}$ (1) and P5 in ethanol with $x_{\mathrm{M}}=1.5 \times 10^{-7}(2)$ [7].

The detailed analysis of the fluorescence spectra of perylene-like dyes in the LB films has been presented in Ref. [7]. It was ascertained that dyes P1-P6 in the LB films can create configurations of molecules giving $Y$-type (excimer precursor) $[20,21]$ and $E$-type (genuine excimer) $[20,22]$ emissions. The contributions of both emission types depend on the length of the alkyl chains substituted to the perylene core, the number of layers and the dye content in the mixture. The alkyl chain length has strong effect on the interaction among aromatic rings in dye molecules and, when the chains are too long, they hinder the creation of the genuine excimers. The increasing number of layers and the dye concentration cause an enhancement of $E$-type emission. The conclusion can be drawn that in these cases, because of a strong interaction among molecules in both ground and excited states, the molecular pairs giving $Y$-type emission relax further to yield such configurations of molecules that emit genuine excimer emission.

In the case of derivatives of 4-aminonaphthalimide the shift and the broadening of the fluorescence band of the dyes in the LB films with respect to that in diluted solutions are also observed. The shift is equal to $15-58 \mathrm{~nm}$, depending on the substituent molecular structure [9]. Figure 9 shows the fluorescence spectra of AN2/7CB mixtures in the LB films for various molar fractions of the dye. It is seen that the position of the band maximum depends on the dye concentration. The content of the dye in the mixture affects also strongly the fluorescence intensity: up to some $x_{\mathrm{M}}$ the fluorescence intensity increases, and next decreases. Such behaviour is observed for all the dyes from this group, although the value at which the fluorescence starts to decrease is different for various dyes. The fluorescence quenching with the rise of the dye concentration is characteristic for the formation of dimers in the excited state $[3,17]$. Because in the absorption spectrum such large band shift as in the fluorescence spectrum is not observed, it can be supposed that dyes AN1-AN6 in the LB films are able to create the excimers or their precursors, similarly as it was found for

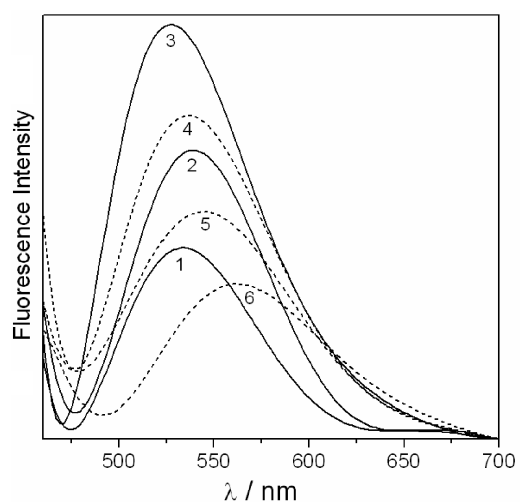

Fig. 9. Fluorescence spectra of $\mathrm{AN} 2 / 7 \mathrm{CB}$ mixtures in LB films; the molar fraction of dye $x_{\mathrm{M}}=0.1$ (1), 0.2 (2), $0.3(3), 0.4(4), 0.6(5)$, and $0.8(6)[9]$.
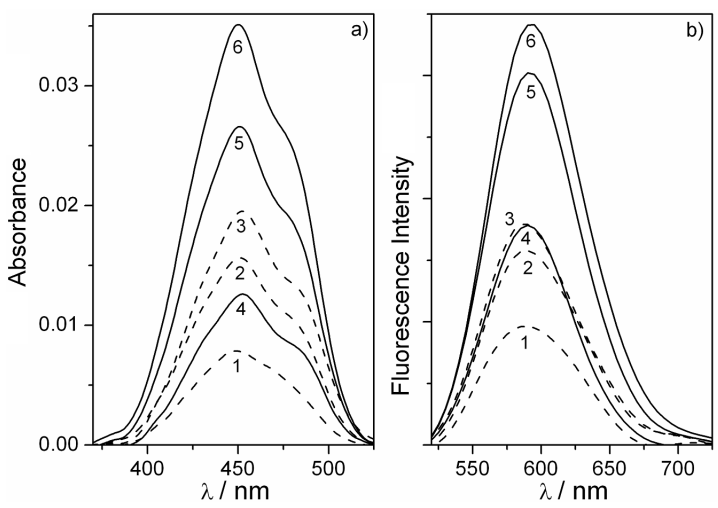

Fig. 10. Absorption (a) and fluorescence (b) spectra of NB1/AA mixture with $x_{\mathrm{M}}=0.5$ in LB films of $Z$-type of 1 (1), $2(2)$, and 3 (3) layers and of $Y$-type of $2(4)$, $4(5)$, and 6 (6) layers [10].

perylene-like dyes. The results of the recent study [23] seem to confirm this suggestion.

The fluorescence spectra of derivatives of naphthoylenebenzimidazole, similarly as $3,4,9,10-$ -tetra-( $n$-alkoxy-carbonyl)-perylenes and derivatives of 4-aminonaphthalimide, show the broadening of the band in the LB films when one compares with the band in diluted solutions. The bathochromic shift of the band, up to $112 \mathrm{~nm}$, is observed, too. As in this case such large band shift of the absorption band was also not found, it is reasonable to assume that dyes NB1-NB3 create excimers in the excited state. The analysis of the results obtained [10] reveals that the genuine excimers occur in the LB films already at $x_{\mathrm{M}} \approx 0.2$. The further increase of the dye content in the mixture with AA as well as the change of the surface pressure at which the transfer of the floating monolayer on the quartz took place, do not influence the shape and the position of the fluorescence band. However, it was observed that with the rise of the deposited layer number, the fluorescence intensity increases, but not proportionally to the absorbance 
changes. This effect can be explained in terms of the creation of different types of aggregates in the ground and excited states. The fluorescence intensity depends also on the way of the deposition of the monolayer on the solid substrate. The arrangement of the dye molecules in the multilayer LB films of $Y$-type [1] is different than that in the multilayers of $Z$-type [1]. This is illustrated in Fig. 10 for NB1/AA mixture.

\section{Summary}

The most of the fluorescent dyes under investigation (perylene-like dyes, derivatives of 4-aminonaphthalimide, derivatives of naphthoylenebenzimidazole) is able to form compressible and stable monolayers at the air-water interface. Azo dyes can create Langmuir films only when they are mixed with a compound playing the role of the supporting matrix. In this study thermotropic liquid crystals from $n \mathrm{CB}$ homologous series $(7 \mathrm{CB}, 8 \mathrm{CB})$ and arachidic acid were used. The molecular organization in the Langmuir films formed of the dyes themselves and of their mixtures with the liquid crystal or arachidic acid was determined.

Absorption spectra in situ for Langmuir films and both absorption and fluorescence spectra for LB films were recorded. It was found that all the dyes studied can create some fraction of the molecular aggregates in the ground electronic state. Perylene-like dyes and derivatives of 4 -aminonaphthalimide create $J$-type aggregates, whereas derivatives of naphthoylenebenzimidazole can form also $I$-type aggregates. Azo dyes are able to form $J$-type and $H$-type aggregates, depending on the concentration. In the excited state the fluorescent dyes can create excimers or their precursors. As a result, the fluorescence intensity of perylene-like dyes and derivatives of 4 -aminonaphthalimide decreases. Opposite behaviour is observed for derivatives of naphthoylenebenzimidazole in LB films. Here the fluorescence intensity increases with the dye concentration and the layer number. This fact causes that the later dyes are the most promising candidates to use them in active layers in OLEDs.

\section{Acknowledgments}

Author wishes to thank Mr. Marek Weiss for his technical assistance.

\section{References}

[1] G. Roberts, Langmuir-Blodgett Films, Plenum Press, New York 1990.
[2] I.H. Stapff, V. Stümpflen, H. Wendorff, D.B. Spohn, D. Möbius, Liq. Cryst. 23, 613 (1997).

[3] A. Kawski, Photoluminescence of solutions, PWN, Warszawa 1992 (in Polish).

[4] Organic Molecular Aggregates: Electronic Excitation and Interaction Processes, Eds. P. Reineker, H. Haken, H.C. Wold, Springer, Berlin 1983.

[5] D. Janietz, in: Handbook of Surfaces and Interfaces of Materials, Vol. 1, Eds. H.S. Nalwa, Academic Press, London 2001, p. 423.

[6] G.L. Gaines, Jr., Insoluble Monolayers at Liquid-Gas Interfaces, Interscience Publishers, New York 1966.

[7] R. Hertmanowski, T. Martyński, R. Stolarski, D. Bauman, Opto-Electron. Rev. 16, 237 (2008).

[8] K. Inglot, A. Kaleta, T. Martyński, D. Bauman, Dyes Pigments 77, 303 (2008).

[9] N. Bielejewska, R. Stolarski, D. Bauman, Z. Naturforsch. A 64, 492 (2009).

[10] D. Bauman, J. Bruździński, R. Hertmanowski, A. Buczek, Spectrochim. Acta A 73, 668 (2009).

[11] D. Bauman, A. Płóciennik, K. Inglot, Acta Phys. Pol. A 115, 203 (2009).

[12] R. Hertmanowski, Ł. Chudziński, T. Martyński, P. Stempniewicz, E. Wolarz, D. Bauman, Liq. Cryst. 31, 791 (2004).

[13] A. Biadasz, K. Łabuszewska, E. Chrzumnicka, E. Michałowski, T. Martyński, D. Bauman, Dyes Pigments 74, 598 (2007).

[14] M. Kasha, H.R. Rawls, M. Ashraf El-Bayoumi, Pure Appl. Chem. 11, 371 (1965).

[15] D. Moebius, Adv. Mater. 7, 437 (1995).

[16] A. Miyata, D. Heard, Y. Unuma, Y. Higashigaki, Thin Solid Films 210/211, 175 (1992).

[17] B. Stevens, M.J. Ban, Trans. Faraday Soc. 60, 1515 (1964).

[18] E. Johnson, R. Aroca, Y. Nagao, J. Phys. Chem. 95, 8840 (1991).

[19] M.I. Sluch, A.G. Vitukhnovsky, Lu Ande, Thin Solid Films 248, 230 (1994).

[20] J. Ferguson, J. Chem. Phys. 44, 2677 (1966).

[21] Z. Salamon, H. Baessler, Chem. Phys. 100, 393 (1985).

[22] S.A. Benning, H.-S. Kitzerow, H. Bock, M.-F. Achard, Liq. Cryst. 27, 901 (2000).

[23] N. Bielejewska, E. Chrzumnicka, R. Stolarski, D. Bauman, Opto-Electron. Rev., DOI:10.2478/s11772-0100002-2. 